Full-length article

\title{
Gambogic acid induces death inducer-obliterator 1-mediated apoptosis in Jurkat T cells
}

\author{
Yong WANG, Yan $\mathrm{CHEN}^{1}$, Zi CHEN, Qing WU, Wen-juan KE, Qiu-ling WU \\ Institute of Hematology, Union Hospital, Huazhong University of Science and Technology, Wuhan 430022, China
}

\author{
Key words \\ gambogic acid; apoptosis; death inducer- \\ obliterator $1 ; \mathrm{NF}-\kappa \mathrm{B}$ \\ ${ }^{1}$ Correspondence to Prof Yan CHEN. \\ Phn 86-27-8572-6387. \\ Fax 86-27-8548-5531. \\ E-mail yanchen@public.wh.hb.cn \\ Received 2007-08-27 \\ Accepted 2007-10-31
}

doi: $10.1111 / \mathrm{j} .1745-7254.2008 .00762 . \mathrm{x}$

\begin{abstract}
Aim: To explore the anticancer effects and the molecular mechanisms of gambogic acid (GA) on Jurkat cells. Methods: Cell viability was assessed by 3-(4,5-dimethylthiazol-2-yl)-2,5-diphenyltetrazolium bromide assay. Annexin V-fluorescein-isothiocyanate/propidium iodide, DNA defragmentation, and comet assay were used to detect apoptosis. Western blotting was used to study the expression of death inducer-obliterator-1 (DIO-1), Bcl-2, NF- $\kappa \mathrm{B}$, and procaspase 3, as well as 2 activated subunits: p17 and p20. The subcellular localization of DIO-1 was examined by immunofluorescence and Hoechst 33258 staining. Results: GA inhibited the proliferation of Jurkat cells with $50 \%$ inhibitory concentration values of $1.51 \pm 0.09(24 \mathrm{~h}), 0.98 \pm 0.13(48 \mathrm{~h})$, and $0.67 \pm$ $0.12 \mu \mathrm{mol} / \mathrm{L}(72 \mathrm{~h})$. GA was able to induce apoptosis of Jurkat cells. Treated by GA, the expression of DIO-1 was upregulated, and that of Bcl-2 and NF- $\kappa \mathrm{B}$ was downregulated, leading to the activation of pro-caspase 3. GA induced the translocation of DIO-1 to the nucleus. Conclusion: GA suppressed the proliferation of Jurkat cells by apoptosis induction. DIO-1 triggered early-stage cell death in GA-treated Jurkat cells.
\end{abstract}

\section{Introduction}

The percentage of long-term remitters and survivors in adult acute lymphoblastic leukemia has not improved significantly during recent decades, although several trials have attempted to intensify the induction and postremission strategy, including early bone marrow transplantation. New drug exploitation is one of the effective options. Gambogic acid (GA), an extraction from the resin of Garcinia hanburyi, was used as folk medicine and a colorant in China. Recently, it has been demonstrated that GA has strong antitumor activities in many solid tumors, such as human hepatoma ${ }^{[1,2]}$, gastric adenocarcinoma ${ }^{[3]}$, lung carcinoma $^{[4]}$, and breast cancer ${ }^{[5]}$. Although the mechanism is not fully understood, it has been found to block the cell cycle $^{[7]}$, induce apoptosis ${ }^{[1-6]}$, and inhibit the gene expression of human telomerase reverse transcriptase ${ }^{[4,6,8,9]}$.

Death inducer-obliterator 1 (DIO-1) is identified as a gene upregulated early in apoptosis by several stimuli. The overexpression of DIO-1 in cells induced massive apop- tosis without any apoptotic stimuli ${ }^{[10]}$. Its predicted amino acid sequence consists of a glutamine-rich region, an acidic sequence, and a canonical bipartite nuclear localization signal (NLS) in the N-terminal region, 2 Zn-finger motifs in the central region, and a C-terminal lysine-rich sequence ${ }^{[10]}$. In healthy cells, DIO-1 was located in the cytoplasm. After overexpression or apoptosis induction, DIO-1 translocates to the nucleus and induces apoptosis by caspase activation. A NLS deletion mutant of DIO-1 (DIO-1 $1 \Delta \mathrm{NLS}$ ) is unable to translocate to the nucleus and upregulates pro-caspase levels or triggers cell death ${ }^{[11]}$. The human Dio- 1 gene is mapped to the long arm of chromosome 20. DIO-1 expression abnormalities have been found in $100 \%$ of human myelodysplastic/myeloproliferative diseases (MDS/MPD) and other myeloid neoplasm patients, but not in lymphoid neoplasm patients or healthy donors. Here, we explored the inhibition effect of GA on Jurkat cells and the reasonable mechanism ${ }^{[12]}$. 


\section{Materials and methods}

Cells and reagents The Jurkat cells were obtained from the China Center for Typical Culture Collection (Wuhan, China) and cultured in RPMI-1640 medium (Gibco, Grand Island, NY, USA) supplemented with 10\% fetal bovine serum (Gibco, USA) at $37{ }^{\circ} \mathrm{C}$ in a humidified atmosphere of $5 \% \mathrm{CO}_{2}$ and $95 \%$ air. GA (Figure 1) was provided by Calbiochem (Darmstadt, Germany). 3-(4,5-dimethylthiazol-2-yl)-2,5-diphenyltetrazolium bromide (MTT) was bought from Janssen Chimica (New Brunswick, NJ, USA). Hoechst33258, DMSO, and monoclonal and mouse anti- $\gamma$-tubulin antiserum were purchased from Sigma-Aldrich (St Louis, MO, USA). Monoclonal rabbit anti-DIO-1 and anticaspase 3 antiserum, monoclonal mouse anti-Bcl-2, and NF- $\mathrm{KB}$ antiserum were the products of Santa Cruz Biotechnology (Santa Cruz, CA, USA). Enhanced chemiluminescence reagent kits were purchased

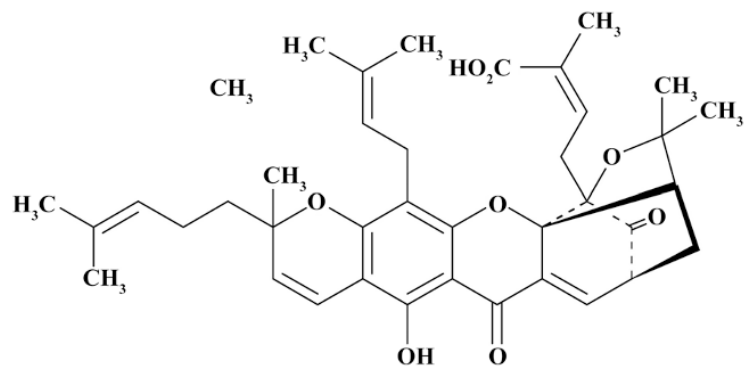

Figure 1. The structure of gambogic acid.

from Pierce Biotechnology (Rockford, IL, USA). Annexin V-fluorescein-isothiocyanate (FITC)/propidium iodide (PI) was from Keygen (Nanjing, China). The Genomic DNA purification kit was bought from Fermentas (Burlington, Canada).

Cytotoxicity assay of GA Cell proliferation was assessed by MTT assay. Briefly, logarithmically-growing Jurkat cells were seeded in triplicate at a concentration of $5 \times 10^{4}$ cells $/ \mathrm{mL}$ in culture medium, and then exposed to various concentrations $(0,0.25,0.5,0.75,1.0,1.5,2.0,3.0$, and $6.0 \mu \mathrm{mol} / \mathrm{L}$ ) and exposure intervals (up to $3 \mathrm{~d}$ ) of GA. Then, the MTT working solution was added to each well and the cells were incubated for $4 \mathrm{~h}$. The water-insoluble formazan was formed during incubation and was solubilized by adding DMSO to each well. Suspension culture growth inhibition and the 50\% inhibitory concentration values $\left(\mathrm{IC}_{50}\right)$ for $\mathrm{GA}$ were determined as previously described $^{[12]}$.

Apoptosis assessment For the Annexin V-FITC/PI as- say, after GA treatment $(0,1,2$, and $3 \mu \mathrm{mol} / \mathrm{L}), 5 \times 10^{5}$ cells were washed in phosphate-buffered saline (PBS) and resuspended in $500 \mu \mathrm{L} 1 \times$ binding buffer. In total, $5 \mu \mathrm{L}$ Annexin V-FITC and $5 \mu \mathrm{L}$ PI were then added into the buffer. After 15-min incubation at room temperature in the dark, the cells were analyzed by flow cytometry. Annexin V-FITC and PI fluorescence was detected in the FL-1 (green) and FL-2 (red) channels, respectively, after the correction to the spectral overlap between the 2 channels was made. Data were analyzed by CellQuest software (Becton Dickinson, San Jose, CA, USA).

For the DNA fragmentation assay, the Jurkat cells were treated with $0,1,2,3$, and $4 \mu \mathrm{mol} / \mathrm{L}$ GA for $24 \mathrm{~h}$, as well as 0 and $2 \mu \mathrm{mol} / \mathrm{L}$ GA for $24 \mathrm{~h}$ and $48 \mathrm{~h}$, respectively. DNA was extracted by the Genomic DNA purification kit, and then the extraction was electrophoresed on a $1.5 \%$ agarose gel and observed by ethidium bromide (EB) staining using the Gel-Pro analyzer (Peiqing Technology, Shanghai, China). For the comet assay, the Jurkat cells were exposed to 0 or $2 \mu \mathrm{mol} / \mathrm{L} \mathrm{GA}$ for $24 \mathrm{~h}$, and then embedded in agarose on microscope slides, lysed, and electrophoresed. DNA fragments caused by single- or double-stranded breaks migrated faster than intact DNA. DNA were stained with PI. The DNA fragments were visible as comet tails by fluorescence microscopy.

Western blotting The Jurkat cells were treated by 0 , $0.5,1.0,2.0$, and $4.0 \mu \mathrm{mol} / \mathrm{L}$ GA for $24 \mathrm{~h}$ or 0,2 , and 4 $\mu \mathrm{mol} / \mathrm{L}$ GA for $2 \mathrm{~h}$. The harvested cells were homogenized respectively in the homogenization buffer, containing protease inhibitor cocktail (1:1000) in $50 \mathrm{mmol} / \mathrm{L}$ Tris, $\mathrm{pH} 7.0$, $1 \mathrm{mmol} / \mathrm{L}$ EDTA, and $1 \mathrm{mmol} / \mathrm{L}$ phenylmethylsulfonyl fluoride. The raw homogenate was centrifuged at $4{ }^{\circ} \mathrm{C}$ for 20 $\min$ at $13400 \times g$. The supernatants $(20 \mu \mathrm{g})$ with $5 \times$ loading buffer were heated for $10 \mathrm{~min}$ at $100{ }^{\circ} \mathrm{C}$ and then loaded onto $10 \%-15 \%$ SDS-PAGE for proteins with different molecule weights. The proteins were electrophoresed and then transferred onto a nitrocellulose membrane. The membrane was blocked with 5\% non-fat dry milk for $1 \mathrm{~h}$ and subsequently incubated overnight with monoclonal rabbit antiDIO-1 and anticaspase 3 antiserum (1:500), monoclonal mouse anti-Bcl-2, and NF- $\mathrm{B}$ antiserum (1:500), as well as anti- $\gamma$-tubulin antiserum $(1: 10000)$. The proteins were detected by using horseradish peroxidase-conjugated goat antirabbit and antimouse secondary antibodies (1:5000), visualized by using a chemiluminescent substrate kit, and exposed to medical X-ray film. The intensity of the blots was quantified with a gel image analyzer (JS380, Peiqing Science and Technology, Shanghai, China). 


\section{DIO-1 immunofluorescence and Hoechst33258} double staining For detecting the nucleus translocation of DIO- $1,5 \times 10^{6}$ Jurkat cells were treated with $4 \mu \mathrm{mol} / \mathrm{L}$ GA for $24 \mathrm{~h}$. After fixing in $4 \%$ paraformaldehyde in $0.1 \mathrm{~mol} /$ L phosphate buffer $(\mathrm{PB})(\mathrm{pH} 7.3)$ at $4{ }^{\circ} \mathrm{C}$, the cells were plated on glass coverslips in a 24 -well plate on the day before the experiment. Briefly, the staining procedure was performed at room temperature in the dark: (1) blocked and permeabilized in the blocking solution containing 3\% bovine serum albumin, $2 \%$ normal goat serum, and $1 \%$ Triton X-100 in PBS for $30 \mathrm{~min}$; (2) incubated with rabbit antiDIO-1 antiserum (1:100) in blocking solution for $24 \mathrm{~h}$; (3) incubated with FITC-conjugated donkey antirabbit immu-

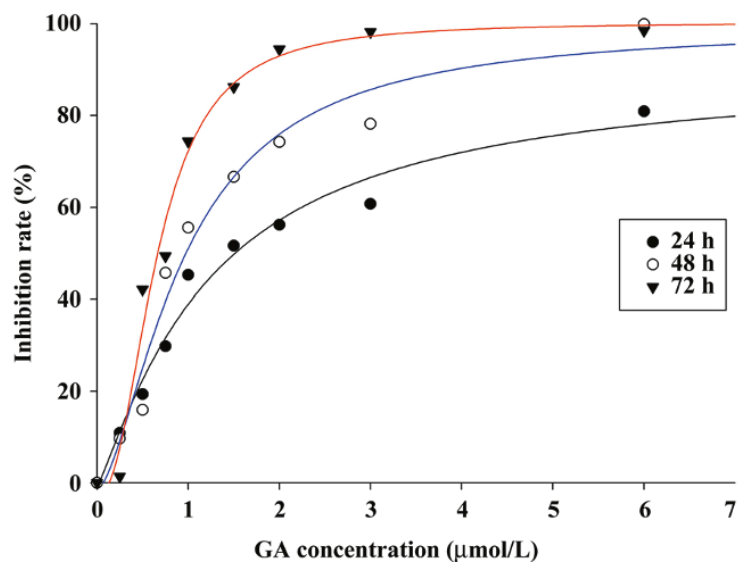

Figure 2. MTT assay of GA-treated Jurkat cells.GA inhibited the proliferation of Jurkat cells in a time and dose-dependent manner. The $\mathrm{IC}_{50}$ values for $24 \mathrm{~h}, 48 \mathrm{~h}$, and $72 \mathrm{~h}$ were $1.51 \pm 0.09 \mu \mathrm{mol} / \mathrm{L}, 0.98 \pm 0.13 \mu \mathrm{mol} / \mathrm{L}$, and $0.67 \pm 0.12 \mu \mathrm{mol} / \mathrm{L}(P<0.01)$, respectively. Curve corresponding to each of the three time intervals $(24 \mathrm{~h}, 48 \mathrm{~h}$, and $72 \mathrm{~h}$ ) was regressed by four parameter logistic equation using Sigmaplot 10.0. noglobulin $\mathrm{G}$ antibody (1:500) in blocking solution for $3 \mathrm{~h}$; and (4) incubated with $10 \mu \mathrm{g} / \mathrm{L}$ Hoechst33258 by the end of step 3 for $15 \mathrm{~min}$. The immunofluorescence was finally visualized in a laser scanning confocal microscope (FV500, Olympus, Tokyo, Japan).

\section{Results}

GA declined proliferation of Jurkat cells The effects of GA on Jurkat cell proliferation were assessed by MTT assay (Figure 2). GA was able to suppress the proliferation of Jurkat cell in a dose- and time-dependent manner. The $\mathrm{IC}_{50}$ values for GA at each interval $(24,48$, and $72 \mathrm{~h})$ were $1.51 \pm 0.09,0.98 \pm 0.13$, and $0.67 \pm 0.12 \mu \mathrm{mol} / \mathrm{L}(P<0.01)$, respectively. However, the time dependence was not significant when the GA concentration was very low or very high.

GA-induced apoptosis of Jurkat cells To clarify the mechanism of GA-induced proliferation inhibition, we examined the GA-induced apoptosis in Jurkat cells. In the Annexin V-FITC/PI assay (Figure 3A-3D), we found that a lower dose of GA (less than $1 \mu \mathrm{mol} / \mathrm{L}$ ) made no difference. The percentages of apoptosis and necrosis cells were less than $4 \%$. However, a higher dose $(2$ and $3 \mu \mathrm{mol} / \mathrm{L})$ of GA treatment in the Jurkat cells resulted in a markedly increased accumulation of apoptotic cells. DNA defragmentation (Figure 3E,3F) and comet assay (Figure 3G, 3H) was designated to analyze the DNA changes in late apoptosis. After incubation with GA, the Jurkat cells induced apoptosis, as shown by the formation of distinct internucleosomal DNA fragmentation, in a dose- and time-dependent manner. In the comet assay, the cells with DNA damage exhibited an oval comet tail.

GA-triggered caspase activation associated with DIO-1 upregulation and translocation DIO-1 was re-
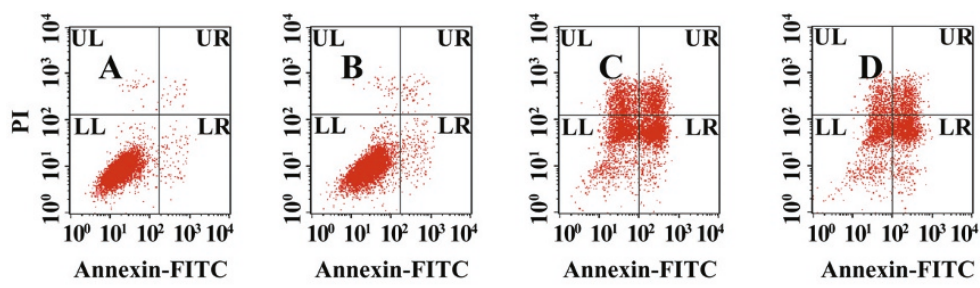

E

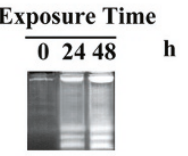

F

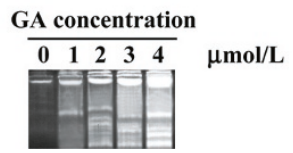

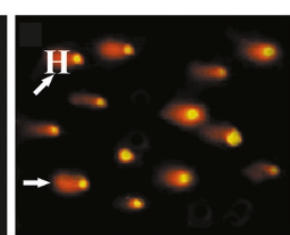

Figure 3. Apoptosis Assessment of GA-treated Jurkat Cells.In Annexin-V/PI staining (A to D), the dual parameter dot plots combining Annexin V-FITC and PI fluorescence show the vial cell population in the lower left quadrant (Annexin V-PI-), the necrotic cells or the damaged cells during the isolation in the upper left quadrant (Annexin V-PI+), the early apoptotic cells in the lower right quadrant (Annexin $\mathrm{V}+\mathrm{PI}-$ ), and the late apoptotic cells in the upper right quadrant (Annexin $\mathrm{V}+\mathrm{PI}+$ ). Treated with 0 (A), 1.0 (B), 2.0 (C), and 3.0 (D) $\mu \mathrm{mol} / \mathrm{L}$ GA for $24 \mathrm{~h}$, percentages of the early apoptotic cells in the whole population was from $0.95 \%$ and $2.09 \%$ to $34.03 \%$ and $38.30 \%$, respectively. (E and F) Dose and time dependent DNA defragmentation. (G and $\mathrm{H}$ ) Dose-dependent DNA electrophoretic mobility. Late apoptotic cells exhibited an oval comet tail (arrow). 
A

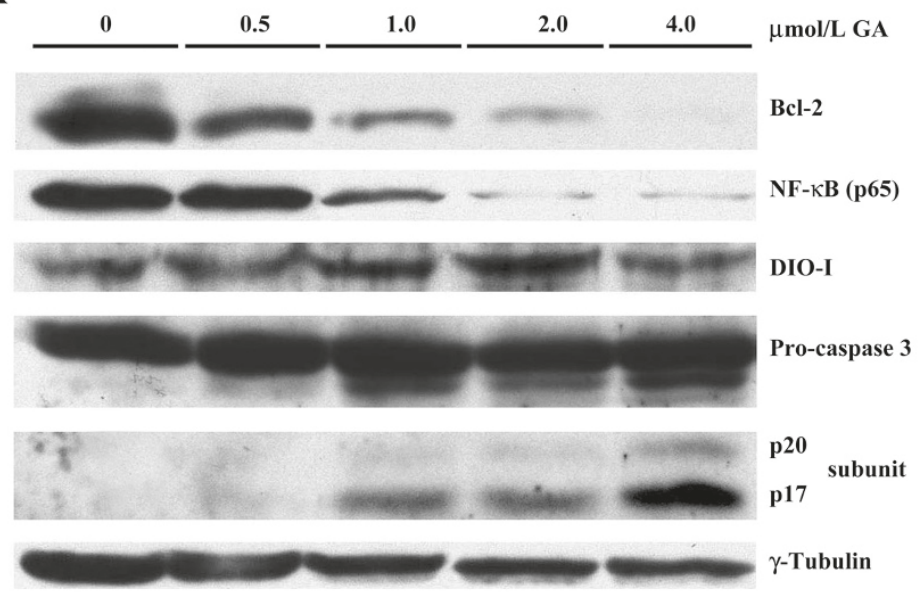

B

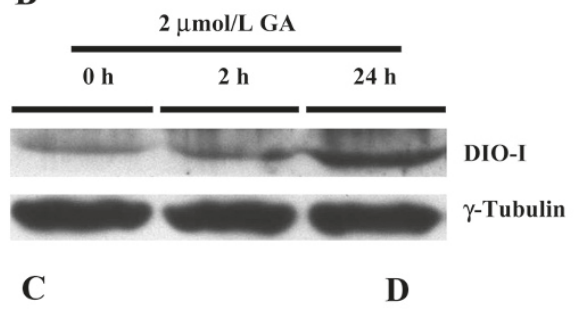

C

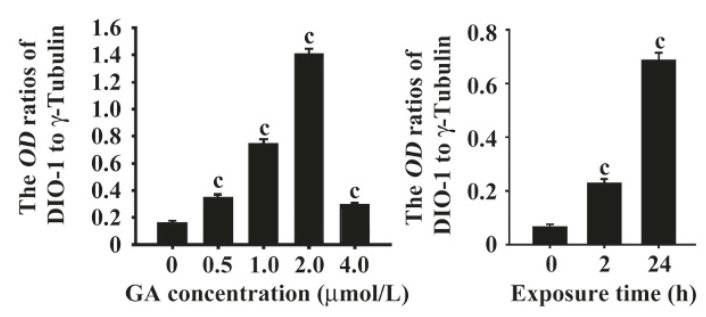

Figure 4. GA-induced apoptosis was mediated by DIO-1. (A)Dose-dependent downregulation of NF-אB and Bcl-2, upregulation of DIO-1, and cleavage of pro-Caspase 3 as well as activated p17 and p20 subuints. (B) Time-dependent upregulation of DIO-1. (C, D) The OD ratios of DIO-1 to $\gamma$-Tubulin for dose-dependent and time-dependent upregulation of DIO-1 ( $\left.{ }^{\mathrm{C}} P<0.01\right)$.

ported to be able to trigger the apoptotic process by caspase activation in vitro and was involved in hematological myeloid neoplasm. However, in our research, we also found DIO-1 expression in lymphoid cells by Western blotting and immunofluorescence. By Western blotting, we detected a dose- and time-dependent increase of DIO-1 expression in Jurkat cells (Figure 4A-4D). The dose-dependent increase of DIO-1 expression was accompanied by the activation of pro-caspase. Pro-caspase was cleaved into 2 activated subunits: p17 and p20 (Figure 4A).

The subcellular localization of DIO-1 was examined by immunofluorescence. Although the nucleus of control cells also slightly exhibited green fluorescence of DIO-1, DIO-1 in the nucleus, if any, was scattered and the nucleus per se was intact (Figure 5A, 5C, 5E, 5G). Treated with $4 \mu \mathrm{mol} / \mathrm{L}$ GA for $24 \mathrm{~h}$, DIO-1 formed a nuclear aggregate in each early apoptotic cell, which showed relatively intact but condensed chromatins. In late apoptotic cells, the condensation started to disintegrate into similarly dense, smaller particles, and DIO-1 aggregation could not be seen (Figure $5 \mathrm{~B}, 5 \mathrm{D}, 5 \mathrm{~F}, 5 \mathrm{H})$.

GA weakened inhibition of caspase activity related to $\mathbf{N F}-\kappa \mathbf{B}$ and Bcl-2 downregulation We then detected the Bcl-2 and NF- $\mathrm{BB}$ expressions in GA-treated Jurkat cells and demonstrated the dose-dependent decline in both Bcl-2 and NF- $\kappa \mathrm{B}$ expressions (Figure 4A).

\section{Discussion}

DIO-1-induced apoptosis was able to be inhibited by benzyloxycarbonyl-Val-Ala-Asp-fluoromethyl ketone (z-
VAD-fmk), a pan caspase inhibitor ${ }^{[10,11]}$, suggesting that DIO-1-induced apoptosis was caspase dependent. In the present study, we reported that the DIO-1 upregulation and translocation was accompanied by the cleavage of procaspase 3 into 2 activated subunits: p17 and p20, so GA-triggered caspase activation may be associated with DIO-1 upregulation and translocation. However, the underlying molecular mechanisms on nuclear translocation and caspase activation remain unknown. DIO-1 forms homo-oligomers in vivo. The cytosolic form of DIO-1 is phosphorylated on serine/threonine and is predominant in healthy cells, whereas the unphosphorylated nuclear form is found under apoptotic conditions. However, phosphorylation alone is not the basis of the mobility change. Additional modifications may thus be demanded for the DIO-1 translocation ${ }^{[11]}$.

Although the function of DIO-1 was not fully clear, several studies evidenced the transcriptional activities of some domains in the DIO- 1 sequence. DIO- 1 contains a plant homeodomain finger and a transcription factor S-II domain; both domains are usually associated with transcription. It has been also reported that the spen paralog and ortholog C terminal (SPOC) is present in the DIO-1 family of proteins and is essential for normal function ${ }^{[14]}$. The SPOC domain of DIO-1 adopts a similar fold than the SPOC domain of the silencing mediator for retinoid and thyroid receptors (SMRT)/histone deacetylase 1 (HDAC1)associated repressor protein (SHARP), and the 7 strands $\beta$-barrel core is maintained ${ }^{[14]}$. Thus, DIO-1 may share a common function and molecular mechanism with SHARP, which has been identified as a component of transcriptional 

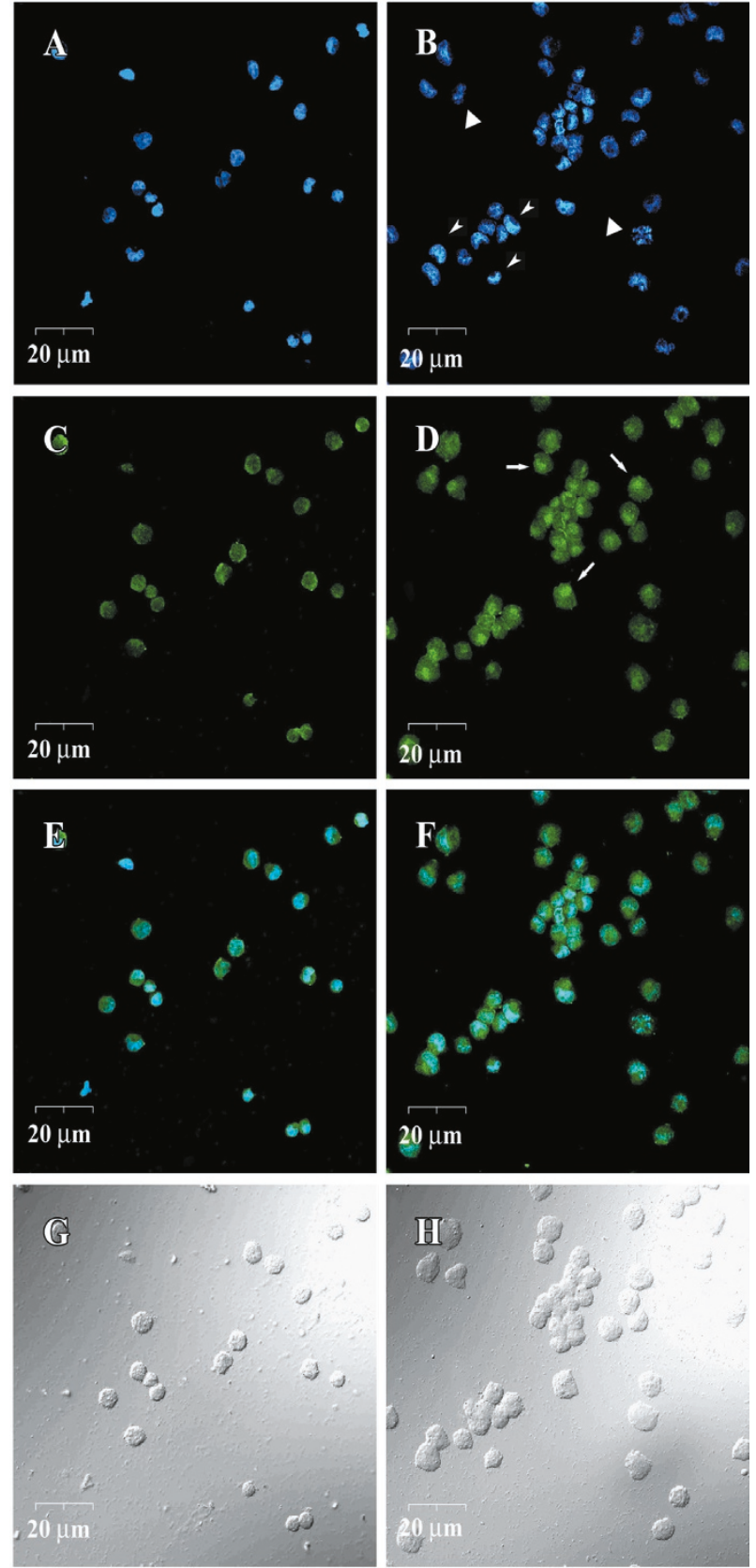

Figure 5. GA induced DIO-1 translocation. A, C, E, and G: $0 \mu \mathrm{mol} / \mathrm{L}$ GA; B, D, F, and H: $4 \mu \mathrm{mol} / \mathrm{L}$ GA; A and B: Hoechst33258-stained chromatins; C and D: FITC-labeled DIO immunofluorescence; E and F: merged image of Hoechst33258 and FITC staining; G and H: bright field image. When treated with $4 \mu \mathrm{mol} / \mathrm{L} \mathrm{GA}$, the nuclear aggregates could be observed (arrow). Some cells showed late apoptotic morphology (triangle), and some represented early apoptotic (arrowhead). The aggregate in late apoptotic cell was not prominent and sometimes invisible.

repression complexes in both nuclear receptor and Notch/ human recombination signal sequence-binding protein $\left(\mathrm{RBP}-\mathrm{J}_{\kappa}\right)$ signaling pathways ${ }^{[15-17]}$. The conserved resi- dues Arg 3552 in the SPOC domain may be important for the protein-protein interaction. Arginine methylation is a common post-translational modification in transcription regulation proteins and is involved in a wide range of cellular processes, including pre-mRNA splicing, polyadenylation, transcription, signal transduction, and cytoskeleton and DNA repair ${ }^{[14]}$. Arginine is also frequently found at the active sites of enzymes, and the SPOC domain may contribute to a specific catalytic function ${ }^{[18]}$. DIO- $1 \Delta$ NLS was unable to translocate to the nucleus or trigger apoptosis ${ }^{[11]}$. The 2 NLS sequences may play an important role in nucleus translocation, recognized by the nucleus transduction components.

In our study, GA induced the downregulation of NF- $\kappa \mathrm{B}$ and Bcl-2. After the activation of NF- $\mathrm{kB}$, its p65 subunit is phosphorylated, leading to nucleus translocation and binding to a specific sequence in DNA, which in turn results in gene transcription, including anti-apoptotic genes (eg Bcl-2, cellular inhibitor of apoptosis protein (cIAP), survivin, and tumor necrosis factor receptor-associated factor (TRAF)); cyclooxygenase-2; matrix metalloproteinase-9; genes encoding adhesion molecules, chemokines, and inflammatory cytokines; and cell cycle regulatory genes (eg cyclin $D$ and $c-M y c)^{[19]}$. In Jurkat cells, NF- $\mathrm{kB}$ is highly expressed and may result in the overexpression of Bcl-2. $\mathrm{Bcl}-2$ is one of the dominant regulators in apoptosis, whose downregulation releases cells from anti-apoptotic status. The overexpression of Bcl-2 blocks DIO-1-induced apopto$\mathrm{sis}^{[10]}$. This indicates that DIO-1 is upstream of the caspase cascade and the induction of apoptosis driven by this gene proceeds through the main apoptotic route. The dosedependent decline in Bcl-2 expression may be mediated by $\mathrm{NF}-\kappa \mathrm{B}$ downregulation. In normal tissues, there must be a balance between the NF- $\kappa \mathrm{B}$-regulated cell proliferation and the DIO-1-mediated apoptosis. However, in tumor tissues, the high expression of NF- $\mathrm{KB}$ expression inevitably suppresses the caspase activity and inhibits DIO-1-mediated apoptosis. We found that GA not only triggered DIO-1mediated caspase activation, but also decreased or eliminated the inhibition of caspase activity in Jurkat $\mathrm{T}$ cells, suggesting that GA anti-leukemia effect was mediated by DIO-1 upregulation and translocation and associated with the caspase-dependent signaling pathway. In summary, it appears that GA as an apoptotic inducer is a potential drug, and DIO-1 as a caspase upstream regulator is a potential target for lymphoblastic leukemia treatment.

\section{Acknowledgements}

We thank Dr He LI, Department of Anatomy, Tongji 
Medical College, Huazhong University of Science and Technology, for the provision of experimental facilities and technical support in the research work. We also thank Wei-xi WANG and Yi-nong ZHANG, technicians from the Division of Histology and Embryology, Tongji Medical College, Huazhong University of Science and Technology, for their technical support in laser confocal microscopy.

\section{References}

1 Gu HY, Guo QL, You QD, Liu W, Qi Q, Zhao L, et al. Gambogic acid induced apoptosis in human hepatoma SMMC27721 cells with p53 and Bax upregulated. Chin J Nat Med 2005; 13: 168-72.

2 Guo QL, You QD, Wu ZQ, Yuan ST, Zhao L. General gambogic acids inhibited proliferation of human hepatoma SMMC-7721 cells in vitro and in nude mice. Acta Pharmacol Sin 2004; 25:769-74.

3 Guo QL, Zhao Li, You QD, Wu ZQ, Gu HY. Gambogic acid inducing apoptosis in human gastric adenocarcinoma SGC-7901 cells. Chin J Nat Med 2004; 12: 106-10.

4 Wu ZQ, Guo QL, You QD, Zhao L, Gu HY. Gambogic acid inhibits proliferation of human lung carcinoma SPC-A1 cells in vivo and in vitro and represses telomerase activity and telomerase reverse transcriptase mRNA expression in the cells. Biol Pharm Bull 2004; 27 : 1769-74.

5 Zhang HZ, Kasibhatla S, Wang Y, Herich J, Guastella J, Tseng B, et al. Discovery, characterization and SAR of gambogic acid as a potent apoptosis inducer by a HTS assay. Bioorg Med Chem 2004; 12: 309_ 17.

6 Yu J, Guo QL, You QD, Lin SS, Zhao L, Gu HY, et al. Repression of telomerase reverse transcriptase mRNA and hTERT promoter by gambogic acid in human gastric carcinoma cells. Cancer Chemother Pharmacol 2006; 58: 434-43.

7 Jun Y, Guo QL, You QD, Zhao L, Gu HY, Yang Y, et al. Gambogic acid induced G2/M phase cell cycle arrest via disturbing CDK7 mediated phosphorylation of $\mathrm{CDC} 2 / \mathrm{P} 34$ in human gastric carcinoma BGC-823 cells. Carcinogenesis 2007; 28: 632-8.

8 Yu J, Guo QL, You QD, Lin SS, Zhao L, Gu HY, et al. Repression of telomerase reverse transcriptase mRNA and hTERT promoter by gambogic acid in human gastric carcinoma cells. Cancer Chemother Pharmacol 2006, 58: 434-43.

9 Guo QL, Lin SS, You QD, Gu HY, Yu J, Zhao L, et al. Inhibition of human telomerase reverse transcriptase gene expression by gambogic acid in human hepatoma SMMC-7721 cells. Life Sci 2006; 78: 1238 -45 .

10 Garcia-Domingo D, Leonardo E, Grandien A, Martinez P, Albar JP, Izpisua-Belmonte JC, et al. DIO-1 is a gene involved in onset of apoptosis in vitro, whose misexpression disrupts limb development. Proc Natl Acad Sci USA 1999; 96: 7992-7.

11 Garcia-Domingo D, Ramirez D, Buitrago GG, Martinez-A C. Death inducer-obliterator 1 triggers apoptosis after nuclear translocation and caspase upregulation. Mol Cell Biol 2003; 23: 3216-25.

12 Futterer A, Campanero MR, Leonardo E, Criado LM, Flores JM, Hernandez JM, et al. Dido gene expression alterations are implicated in the induction of hematological myeloid neoplasms. J Clin Invest 2005; 115: 2351-62.

13 Bhalla K, Hindenburg A, Taub R, Grant S. Isolation and characterization of an anthracycline-resistant human leukemic cell line. Cancer Res 1985; 45: 3657-62.

14 Sánchez-Pulido L, Rojas AM, van Wely KH, Martinez-A C, Valencia A. SPOC: a widely distributed domain associated with cancer, apoptosis and transcription. BMC Bioinformatics 2004; 7: 91.

15 Evans RM. SHARP, an inducible cofactor that integrates nuclear receptor repression and activation. Genes Dev 2001; 15: 1140-51.

16 Oswald F, Kostezka U, Astrahantseff K, Bourteele S, Dillinger K, Zechner U, et al. SHARP is a novel component of the Notch/RBPJkappa signaling pathway. EMBO J 2002; 21: 5417-26.

17 Ariyoshi M, Schwabe JW. A conserved structural motif reveals the essential transcriptional repression function of Spen proteins and their role in developmental signaling. Genes Dev 2003; 17: 1909-20.

18 Boisvert FM, Cote J, Boulanger MC, Richard S. A proteomic analysis of arginine-methylated protein complexes. Mol Cell Proteomics 2003; 2: 1319-30.

19 Aggarwal BB. Nucleus factor-kappaB: the enemy within. Cancer Cell 2004; 6: 203-8. 